\title{
Artéria carótida interna aberrante na orelha média
}

\section{Aberrant internal carotid artery in the middle ear}

\section{Corintho Viana ${ }^{1}$, Fábio Coelho², Silvio Caldas Neto ${ }^{3}$, Kátia Oliveira ${ }^{4}$, Nelson Caldas}

Palavras-chave: orelha média, malformação, artéria carótida interna aberrante.

Key words: middle ear, congenital malformation, aberrant internal carotid artery.

\section{Resumo / Summary}

alformações vasculares na orelha média são raras, e a artéria carótida interna $(\mathrm{ACl})$ aberrante deve ser lembrada no diagnóstico diferencial, visto o grande risco trazido por uma intervenção cirúrgica inadvertidamente realizada na cavidade timpânica. 0 paciente pode apresentar-se assintomático ou, comumente, com hipoacusia e/ou zumbido pulsátil. As imagens fornecidas pela tomografia computadorizada (TC) e ressonância magnética (RM), incluindo a angiorressonância, firmam o diagnóstico. O s autores descrevem um caso em criança de 13 anos, em que o diagnóstico foi feito através de exames de imagens, direcionados por uma importante suspeita clínica.

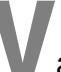

ascular anomalies of the middle ear are uncommon and, because aberrant internal carotid artery, among others, implies great risk at middle ear surgery, it must be remembered at the differential diagnosis. The patient may be asymptomatic, or complains of pulsatile tinnitus and conductive hearing loss. Computer tomography of the temporal bones and magnetic resonance imaging supplemented with magnetic resonance angiography can make the diagnosis. The authors present an aberrant internal carotid artery on a 13-year-old patient, whose diagnosis was performed by otoscopic examination, and imaging methods based on a previous clinical suspicion.
\end{abstract}

\footnotetext{
${ }^{1}$ Médico Residente do Serviço de ORL do HC - UFPE.

${ }^{2}$ Médico Assistente do Serviço de ORL do HC - UFPE.

${ }_{3}^{3}$ Professor Adjunto da Disciplina de ORL do HC - UFPE

${ }^{4}$ Médica Voluntária de Serviço de ORL de HC - UFPE.

5 Professor Titular da Disciplina de ORL do HC - UFPE.

Serviço de Otorrinolaringologia, Hospital das Clínicas, Universidade Federal de Pernambuco.

Endereço para Correspondência: Corintho Viana - Av. Eng. Domingos Ferreira, 3181/403 Boa Viagem Recife PE 51020-035 E-mail: corinthoviana@ig.com.br

Trabalho aprovado para apresentação oral no $36^{\circ}$ Congresso Brasileiro de Otorrinolaringologia, 19 a 23 de novembro de 2002 , Florianópolis - SC

Artigo recebido em 12 de dezembro de 2002. Artigo aceito em 24 de agosto de 2003.
} 


\section{INTRODUÇÃO}

A incidência da artéria carótida interna $(\mathrm{ACl})$ aberrante no espaço da orelha média é de aproximadamente $1 \%$ na população geral, e a grande maioria é assintomática ${ }^{1}$.

Esta anomalia do canal carotídeo, quando não lembrada, pode eventualmente levar a um diagnóstico equivocado de otite média secretora ou de tumor vascular de orelha média, que pode levar a graves acidentes cirúrgicos.

A maioria dos casos dos pacientes sintomáticos apresenta-se com zumbido pulsátil e hipoacusia, podendo ocorrer vertigens, otalgia, plenitude auricular e perda auditiva condutiva progressiva².

0 diagnóstico diferencial da $\mathrm{ACl}$ aberrante na orelha média é feito com: colesteatoma congênito, tumor glômico timpânico, otite secretora, fístula arteriovenosa, fistula do seio cavernoso, fístula carotídea, bulbo jugular deiscente, aneurisma petroso de artéria carótida interna, dilatação de artéria basilar, persistência de artéria basilar, alteração da artéria meníngea média, como é demonstrado na literatura ${ }^{3-5}$.

\section{RELATO DO CASO}

Paciente R.M.G.S.C., 13 anos, sexo feminino, branca, encaminhada ao nosso serviço com história de hipoacusia flutuante e zumbido na orelha esquerda há aproximadamente 6 meses. Um zumbido não pulsátil, tipo chiado.

0 exame otorrinolaringológico mostrou orofaringoscopia, rinoscopia, exame do pescoço e otoscopia da orelha direita sem anormalidades. À oto scopia da orelha esquerda evidenciou-se orelha externa normal e membrana timpânica intacta, porém com opacificação difusa. Durante otomicroscopia, o espéculo utilizado "pulsava" na orelha externa esquerda, acompanhando os batimentos cardíacos da paciente.

À inspeção da paciente evidenciou-se múltiplas lesões eritematosas, de características vasculares, nos membros superiores e face. Passado cirúrgico de duas ressecções de hemangiomas de face, sem outras patologias.

A audiometria apresentou hipoacusia condutiva à esquerda e normoacusia à direita e curva tipo B à imitanciometria do ouvido esquerdo, com movimentação rítmica da agulha do aparelho.

Visando identificar a alteração vascular, que levaria a estes achados clínicos entre tantos diagnósticos diferenciais, solicitou-se uma tomografia computadorizada (TC) dos ossos temporais e ressonância nuclear magnética (RNM) com angiorressonância.

A TC evidenciou velamento da orelha média esquerda, em continuidade com o canal carotídeo intrapetroso (Figura 1).

Os achados relevantes da angiorressonância magnética foram: agenesia da porção vertical cervical da $\mathrm{ACl}$ esquerda, com fluxo através de anastomoses intratimpânicas, artérias carótico-timpânicas e timpânica inferior, formando uma artéria, que comunicava-se com o ramo horizontal da artéria carótida interna esquerda, compatível com um curso aberrante desse vaso. Via-se também ectasia da porção intra-petrosa da artéria (Figura 2).

0 tratamento conservador foi adotado, com orientação da família quanto a sua patologia, alertando-os quanto ao grande risco de uma intervenção cirúrgica nesta orelha.

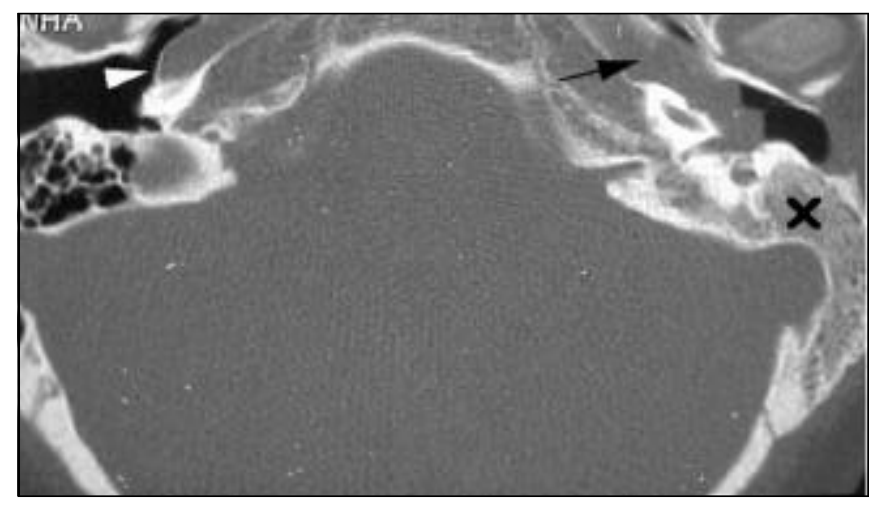

Figura 1. Tomografia axial computadorizada dos ossos temporais mostrando, do lado direito, canal carotídeo íntegro (seta branca). À esquerda, esta parede óssea não existe, havendo continuidade entre o conteúdo do canal carotídeo (seta preta) e a orelha média. A mastóide deste lado é ebúrnea (r), provavelmente por obstrução do pró-tímpano pela carótida aberrante.

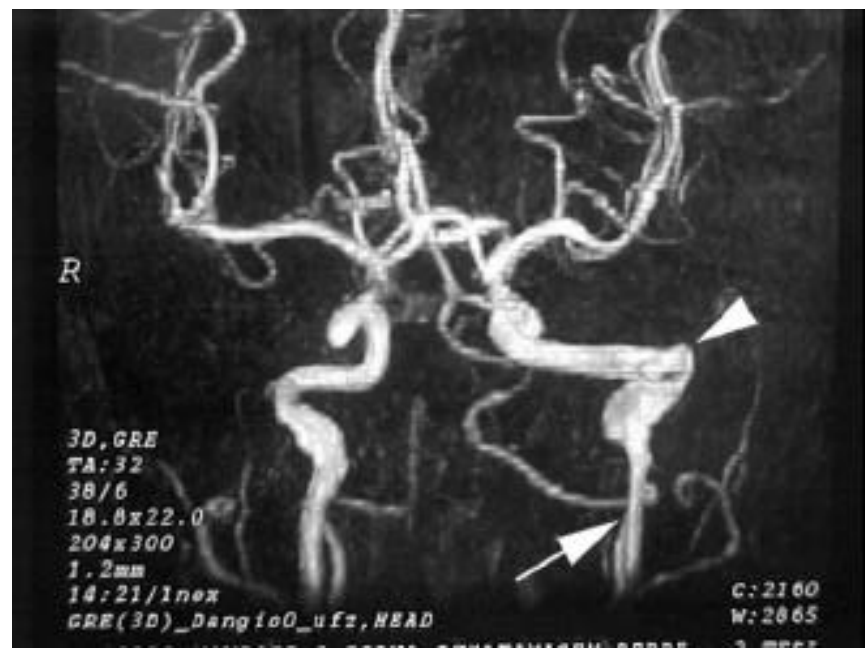

Figura 2. Angiorressonância mostrando agenesia da porção cervica da $\mathrm{ACl}$, substituída por ramos arteriais ascendentes (seta) que alimentam a porção intra-petrosa da artéria ectasiada (cabeça de seta). 


\section{DISCUSSÃO}

Cursos aberrantes de vasos petrosos são extremamente raros ${ }^{1}$. Na maioria dos casos, os pacientes são assintomáticos e quando há sintomas, são compostos, normalmente, de zumbido pulsátil e hipoacusia.

A ACl intratimpânica aparece, principalmente, em mulheres (numa proporção de 5:1 em relação aos homens) e no lado direito (2:1, em relação ao esquerdo) ${ }^{2}$.

A carótida penetra no osso petroso, via canal carotídeo, medialmente ao processo estilóide, separado da veia jugular interna apenas pela bainha carotídea. 0 segmento inicial da $\mathrm{ACl}$ é vertical e anterior à cóclea e separado da orelha média por uma fina camada óssea de $0,5 \mathrm{~mm}$ de espessura em média ${ }^{6}$.

Anomalias da $\mathrm{ACl}$ no osso temporal incluem aneurisma e deslocamento da artéria, que poderiam ocorrer por causas congênitas ou adquiridas. $\mathrm{O}$ curso anormal da $\mathrm{ACl}$ na orelha média pode ser explicado embriologicamente, por uma malformação do primeiro e segundo arcos branquiais, resultando numa persistência de estruturas vasculares embriológicas, vasos e anastomoses, na cavidade timpânica. Isto levaria à não formação da parede óssea que separa a $\mathrm{ACl}$ do ouvido médio?.

No exame otoscópico, pode ser encontrada uma massa vermelha, vista por transparência na metade anterior da orelha média, de característica pulsátil ou não; à audiometria, perda auditiva condutiva na orelha acometida, a imitanciometria ocasionalmente revelará uma membrana timpânica pulsátili.

O s sinais e sintomas clínicos podem, entretanto, não diferenciar a $\mathrm{ACl}$ aberrante de outras anomalias vasculares que podem estar presentes na orelha média. Por isso, 0 diagnóstico deve ser firmado por exames radiológicos complementares, como a angiografia convencional, a TC e a RNM com angiorressonância.

A TC do osso temporal define a organização vascular e a estrutura óssea. $\mathrm{A} \mathrm{ACl}$ aberrante é definida pelos seguintes achados: massa intratimpânica, canalículo timpânico inferior alargado, ausência do segmento vertical da $\mathrm{ACl}$ e da cobertura óssea da porção timpânica da artéria9. A TC pode, entretanto, em alguns casos, não diferenciar a anomalia da $\mathrm{ACl}$ do tumor glômico muito vascularizado, este apresentando-se como massa vascular com parede óssea íntegra ${ }^{10}$.

A angiografia convencional é um método útil para definir anormalidades $\mathrm{da} \mathrm{ACl}$, suprimento vascular, exclusão de aneurisma e planejamento de intervenção cirúrgica. Entretanto, por seu potencial de complicações importantes, em geral, pode ser dispensada em favor dos outros métodos de imagem. Achados semelhantes podem ser demonstrados através da RNM, ajudando a caracterizar e definir a extensão da lesão, valendo-se da angiorressonância, com menor morbidade ${ }^{1}$.

A necessidade de tratamento é controversa nos casos diagnosticados clínica ou radiologicamente; pode ser instituído para resolver os sintomas ou para prevenir complicações, como erosão ossicular ou sangramentos. Neste tipo de situação, o melhor tratamento, segundo Cohen, 1981, e Hunt, 2000, é proibir a manipulação da orelha média e informar os cuidados que este paciente deve ter ${ }^{11,12}$, pois os benefícios deste tipo de procedimento, em geral, não compensam os riscos cirúrgicos e anestésicos.

Ruggles e Reed, 1972, recomendaram a separação da artéria do espaço da orelha média, com enxerto de fáscia e cera de $0 \mathrm{SSO}^{13}$, o que, segundo Valvassori e Buckingham, 1974, pode predispor à formação de aneurisma ${ }^{14}$. Outro método descrito é separar os ossículos da $\mathrm{ACl}$, com retalho de fáscia ou lâmina de silicone, buscando evitar a erosão ossicular8.

Entretanto, na ocorrência de uma lesão inadvertida da $\mathrm{ACl}, 0$ tratamento deve ser instituído para salvar a vida do paciente. A conduta inicial seria a colocação de tampão de surgicel ${ }^{7}$ e algodão na orelha média e no conduto auditivo externo e curativo oclusivo, mantido por várias semanas para evitar a recorrência do sangramento. O utros estágios de terapêutica para controle da hemorragia incluem o tampão nasal posterior, ligadura cirúrgica da $\mathrm{ACl}$, ou a oclusão do vaso por balão $2,12,13$.

\section{COMENTÁRIOSFINAIS}

Alterações vasculares na orelha média, incluindo artéria carótida interna aberrante, devem sempre ser lembrados pelos otorrinolaringologistas, evitando acidentes na manipulação inadvertida da orelha média, por vezes até catastróficos.

As imagens da tomografia computadorizada e da RNM com angiorressonância devem ser realizadas quando da suspeita de uma $\mathrm{ACl}$ aberrante na cavidade timpânica, ajudando no diagnóstico diferencial dessa patologia incomum.

0 tratamento, mesmo necessário em algumas situações, é controverso. Deve ao máximo ser evitado, visto os grandes riscos do procedimento e suas possíveis conseqüências.

\section{REFERÊNCIASBIBUOGRÁFICAS}

1. Botma $M$ et al. Aberrant internal carotid artery in the middle-ear space. J Laryngo Otol. 2000; 114: 784-787;

2. Ridder GJ, Fradis M, Schipper J. Aberrant internal carotid artery in the middle ear. Ann Otol Rhinol Laryngol 2001; 110(9):892-4.

3. Goldman NC, Singleton GT, Holly EH. Aberrant carotid artery presenting as a mass in the middle ear. Arch Otolaryngol 1971; 94:269-73.

4. Bold EL, Wanamaker HH, Hughes GB et al. Magnetic resonance angiography of vascular anomalies of the middle ear. Laryngoscope 1994;104:1404-11.

5. Tian Q, Linthicum FH Jr. Temporal bone histopathology of aberrant carotid artery. Otolaryngol Head Neck Surg 1998; 119:506-7.

6. Glasscock ME III, Dickins JR, Jackson CG, Wiet RJ. Vascular anomalies of the middle ear. Laryngoscope 1980; 90:77-88. 
7. Sinnreich Al, Parisier SC, Cohen NL, Berreby M. Arterial malformatios of the middle ear. Otolaryngol Head Neck Surg 1984; 92:194-206.

8. Cole RD, May JS. Aberrant ICA. South Med J 1994; 87:1277-80.

9. Mcelveen JT Jr, Lo WWM, El Gabri TH, Nigri P. Aberrant ICA: classical findings on computed tomography. Otolaryngol Head Neck Surg 1986; 94:616-21.

10. Remley KB et al. Pulsatile tinnitus and the vascular tympanic membrane: CT, MRI and radiographic findings. Radiology 1990; 174:33-7.

11. Cohen SR, Briant TD. Anomalous course of the internal carotid artery - a warning. J Otolaryngol 1981; 10:283-6.
12. Hunt JT, Andrews TM. Management of aberrant internal carotid artery injuries in children. Am J Otolaryngol 2000; 21:50-4.

13. Ruggles RL, Reed RC. Treatment of aberrant carotid arteries in the middle ear: a report of two cases. Laryngoscope 1972; 82:1199205.

14. Valvassori GE, Buckingham RA. Middle ear masses mimicking glomus tumors radiographic and otoscopic recognition. Laryngoscope 1974; 82:119-205.

15. Glasgold AI, Horrigan WD. The internal carotid artery presenting as middle ear tumor. Laryngoscope 1972; 82:2217-21. 